\title{
MODELLING OF DYNAMICAL BEHAVIOR OF PNEUMATIC SPRING - MASS SYSTEM
}

\author{
T. TranXuan*, D. Cirkl ${ }^{* *}$
}

\begin{abstract}
This article deals with the simulation model of a pneumatic spring element. The purpose of this spring is to be implemented into car seat cushioning and provide a change of pressure distribution in the contact zone between seat and passenger. This function is ensured by a feedback loop controlled electropneumatic circuit. The mathematical model of the system is described by analytical functions which represent mechanical behavior of pneumatic valves, PID controller and used materials. The variable volume of the spring is considered. Dynamical response of the spring-mass system to kinematic excitation is investigated using Matlab software and transfer functions of acceleration are presented in dependence on the level of pressurization of the spring.
\end{abstract}

Keywords: car seat, vibration isolation, polyurethane foam, pneumatic spring, transmission of acceleration

\section{Introduction}

The pneumatic spring element is a part of the system intended to change pressure distribution in the contact zone between the passenger and the seat. Its design is in accordance with a patented solution (Cirkl, 2012) and comprises an electro-pneumatic circuit and spring-mass subsystem. As the system is aimed to use in means of transport it is important to study its dynamical behavior.

\section{Model of the system}

For purpose of creating the simulation model, the system is divided into two main parts in accordance with Fig. 1. The first subsystem is represented by the controlled electro-pneumatic feedback circuit and the second one, the mechanical subsystem, is represented by a PU foam block and by pneumatic spring. Both subsystems are described by analytical functions.

\subsection{Model of the electro-pneumatic feedback circuit}

The pneumatic circuit comprises two kinds of pneumatic valves. The proportional valves denoted $\mathrm{V}_{1}$ and $\mathrm{V}_{2}$ are characterized by the dependence of flow rate on pressure difference $\Delta p$ between input and output pressure, $p_{1}$ and $p_{2}$, respectively, and by input coil current $i$. In the pneumatic circuit, there are also implemented two digital valves $\mathrm{V}_{3}$ and $\mathrm{V}_{4}$. Flow characteristic of the digital valve includes sonic conductance $C=0.083 \mathrm{l} /$ (s.bar) and critical pressure ratio $b=0.28$. Formulas for the calculation of the flow rate are presented in (Cirkl and TranXuan, 2016a).

The pressure inside the reservoir $p_{\text {in }}$ is a parameter which changes in time and influences the system dynamics. According to (Cirkl and TranXuan, 2016) pressure $p_{i n}$ is limited by range $\left[p_{\min }, p_{\max }\right]$ which makes the compressor work just in case of $p_{i n}<p_{\min }=90 \mathrm{kPa}$ and stop in case of $p_{i n}>p_{\max }=110 \mathrm{kPa}$.

Tien Tran Xuan, MsC.: Department of Applied Mechanics, Faculty of Mechanical Engineering, Technical University of Liberec, Studentská 2, 46117 Liberec, CZ, tien.tran.xuan@tul.cz

** Doc. David Cirkl, PhD.: Department of Applied Mechanics, Faculty of Mechanical Engineering, Technical University of Liberec, Studentská 2, 46117 Liberec, CZ, david.cirkl@tul.cz 


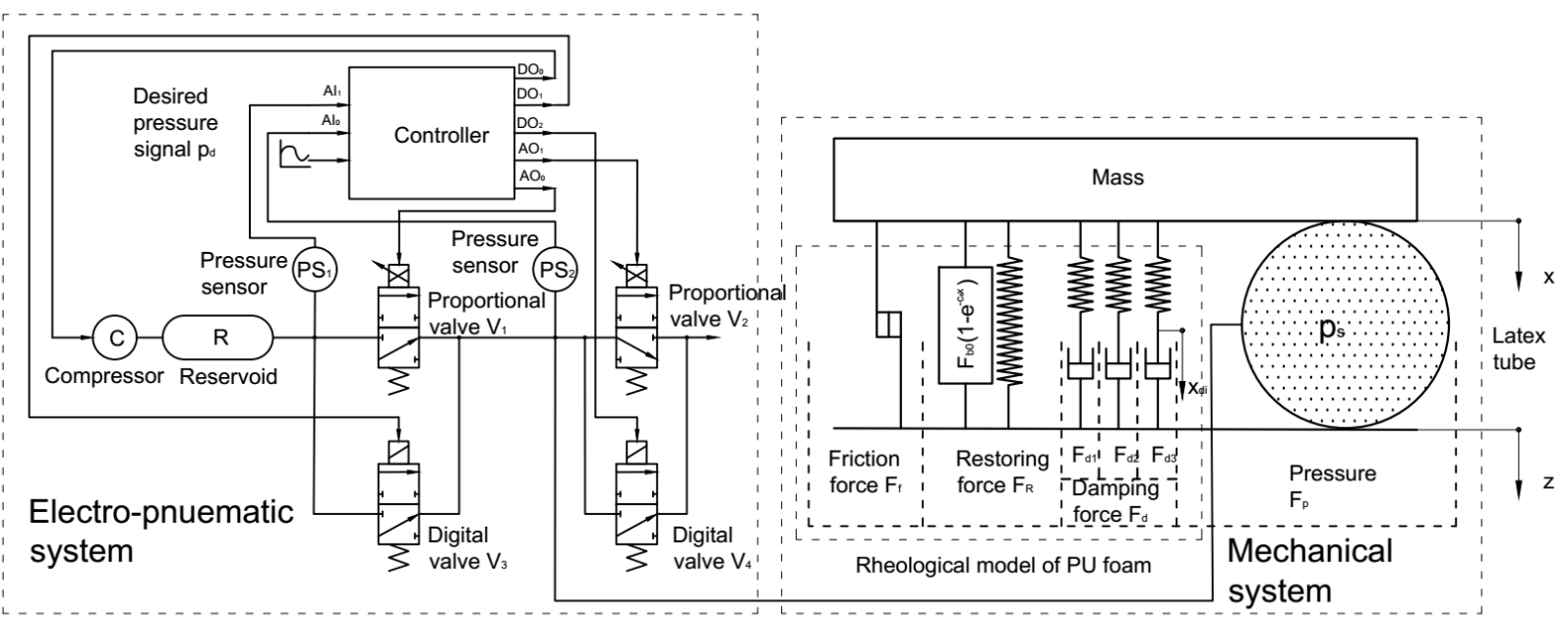

Fig. 1: Scheme of electro-pneumatic system

\subsection{Model of mechanical subsystem}

The model of pneumatic spring element loaded by mass is shown on the right side of Fig.1. The pneumatic spring is made up from cylindrical latex tube filled with PU foam inserted into PU foam cuboidal block. Considering such a structure the pneumatic spring element can be analyzed as a combination of PU foam and latex tube in the parallel arrangement because they have the same displacement $x$ and the same applied excitation $z$.

Computational scheme of the rheological model the PU foam block is depicted in Fig.1. This concept of lumped parameters model follows from the phenomenological approach. It comprises nonlinear restoring force $F_{R}$, damping force $F_{d}$ and frictional force $F_{f}$ in general force response $F_{\text {foam }}$. Model definition is described in detail in (Cirkl and Hrus, 2013).

The scheme of the pneumatic spring element is shown in Fig.2. When it is loaded by a mass the shape of latex tube is deformed therefore volume of latex tube $V$ depends on the displacement $(x-z)$ and the internal pressure $p_{s}$.

As a simplification, it is considered that the volume of the latex tube deforms just at the area of contact with mass (volume $V_{l}$ ) and at two free ends (volume $V_{3}$ ). Volume $V_{2}$ is not in contact with the mass and it is considered as non-deformable. The elliptical cross-section volume $V_{l}$ is considered. Its size depends on the displacement $(x-z)$ :

$$
V_{1}(x-z)=\pi \cdot a \cdot b \cdot l_{1}
$$

where

$a=R-\frac{x-z}{2}, b \approx \sqrt{2 R^{2}-a^{2}}, l_{l}$ is the length of the tube corresponding to the volume $V_{l}\left(l_{l}=0.2 \mathrm{~m}\right), R$ is the latex tube radius before the deformation $(R=0.025 \mathrm{~m})$.

Volume $V_{2}$ is given by the formula:

$$
V_{2}=\pi \cdot R^{2} \cdot l_{2},
$$

where $l_{2}$ is the length of the tube corresponding to the volume $V_{2}\left(l_{2}=0.1 \mathrm{~m}\right)$. According to basic geometry (Fig.3a), the volume of a sphere cap $V_{3}$ with radius $r$ and height $h$ is given by the formula:

$$
V_{3}=\pi(h)^{2} \cdot\left(r-\frac{h}{3}\right)
$$

where $h=l+l_{3}, l_{3}$ is the length of the tube corresponding to the volume $V_{3}\left(l_{3}=0.02 \mathrm{~m}\right), l$ is a displacement of the center point of the free end of the tube and depends on pressure $p_{s}$ which was found 
from experimental data by curve fitting procedure (Fig.3b) where $l$ is considered as a one-parameter function $l=\sum_{i=1}^{15} k_{i} p_{s}$. The experimental arrangement is shown in Fig.3c.

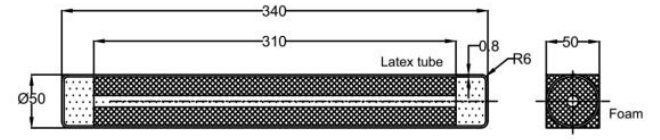

a) Undeformed shape

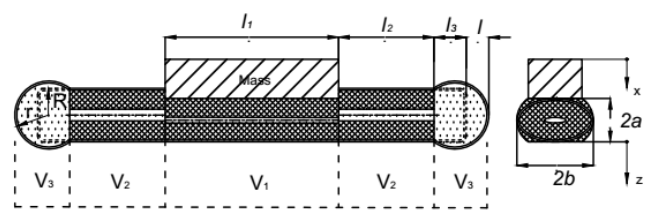

b) Deformed shape

Fig. 2: Scheme of the pneumatic spring element.

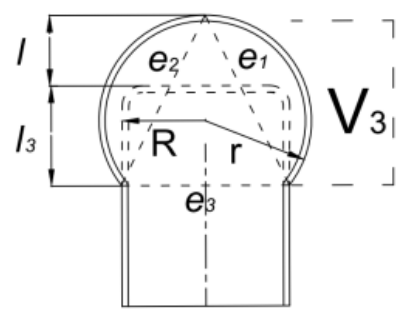

a) Scheme of deformed volume $V_{3}$

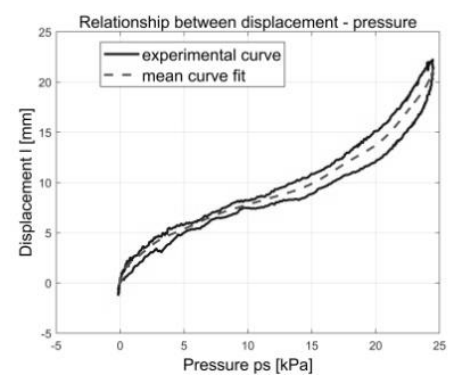

b) Result of experiment

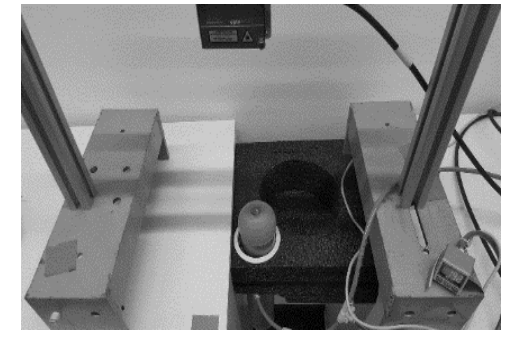

c) Setup of experiment

Fig. 3: The relationship between displacement of the center point at the top side (l)and pressure $\left(p_{s}\right)$.

The radius of partial sphere $r$ is given by the formula:

$$
r=\frac{e_{1} e_{2}}{\sqrt{\left(e_{1}+e_{2}\right)^{2}+e_{3}^{2}}},
$$

where $e_{1}=e_{2}=\sqrt{\left(R^{2}+\left(l+l_{3}\right)^{2}\right)}, \quad e_{3}=2 R$.

The total volume of compressed air in the latex tube is

$$
V=V_{1}+2 V_{2}+2 V_{3}-V_{\text {foam }},
$$

where $V_{\text {foam }}$ is the volume of PU foam that occupies the interior of the latex tube. This value is determined experimentally by the difference between the volume of water before and after soaking the PU foam $\left(V_{\text {foam }}=0.15 \mathrm{~m}^{3}\right)$.

Following (Cirkl and TranXuan, 2016a), pressure $p_{\mathrm{s}}$ is given by differential equation

$$
\dot{p}_{s}=\frac{\kappa \cdot q_{s} \cdot R_{\text {gas }} \cdot T}{V}-\kappa \cdot p_{s} \cdot \frac{\dot{V}}{V}
$$

and force interaction between pneumatic spring element and mass is

$$
F_{p}=S\left(p_{s}-p_{\text {out }}\right) \text {, }
$$

where $T$ is temperature of gas inside cylinder, assumed to be constant $\left(T=297{ }^{0} \mathrm{~K}\right.$ ), $K=1.4$ is adiabatic exponent, $R_{g a s}=287 \mathrm{~J} \mathrm{~kg}^{-1} \mathrm{~K}^{-1}$ is a gas constant, $p_{\text {out }}$ is outside pressure (atmospheric pressure $p_{\text {out }}=0.1 \mathrm{MPa}$ ), $S$ is the contact area between mass and pneumatic spring with considered constant value $\left(S=0.002 \mathrm{~m}^{2}\right)$.

So the equation of motion of the mass is written as:

$$
m . g-F_{\text {foam }}-F_{p}=m . \ddot{x} .
$$




\section{Numerical simulation}

The system of equations derived above makes up the mathematical model of the combined electropneumatic system of spring in interaction with mass. The simulations were performed in Matlab software for two modes of seat operation: a) constant stiffness and b) constant pressure described in more detail in (Cirkl and TranXuan, 2016a). Fig.4a and Fig.4b present simulation results of acceleration transfer function for both control modes mentioned above. In Fig.4c there is presented the system response for idealized case of constant pressure mode (in this case it is assumed that PID controller is able to ensure that pressure $p_{s}$ follows the value of desired pressure $p_{d}$ exactly, it means the error $e=p_{s^{-}} p_{d}=0$ for all time of the process). The displacement excitation $z(t)$ is given by sweep sinus function with modulated amplitude and frequency and constant amplitude of acceleration $0.1 \mathrm{~g}$. The pneumatic spring is loaded by a mass $m=25 \mathrm{~kg}$. Acceleration transfer functions are calculated as a ratio of the amplitude of acceleration of mass and amplitude of acceleration of excitation $\left(A_{x} / A_{z}\right)$. The transfer curves are shown for cases of desired pressure $p_{d} \in\{0,5,10,15,20,25\} \mathrm{kPa}$ in dependence on frequency of excitation in the range $[1,11] \mathrm{Hz}$.

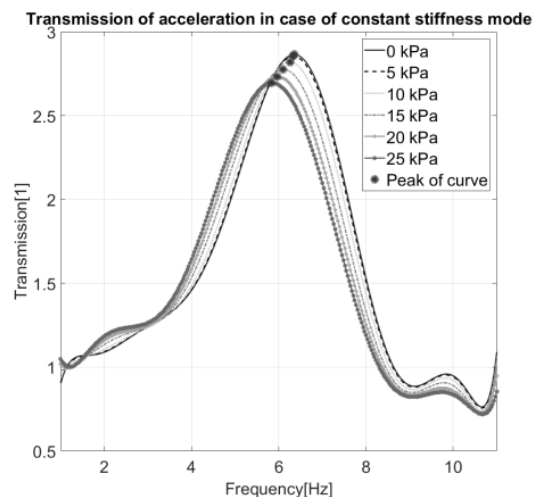

a) Real constant stiffness mode

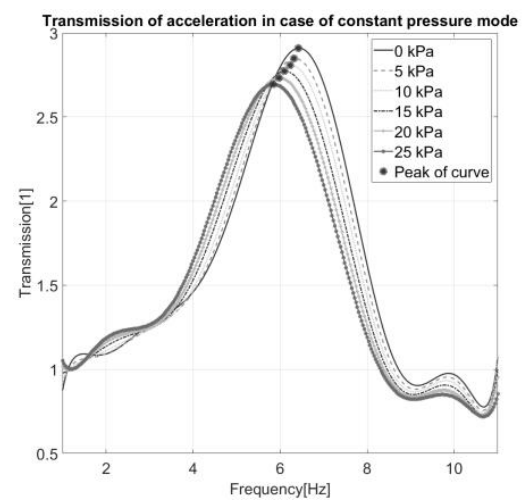

b) Real constant pressure mode

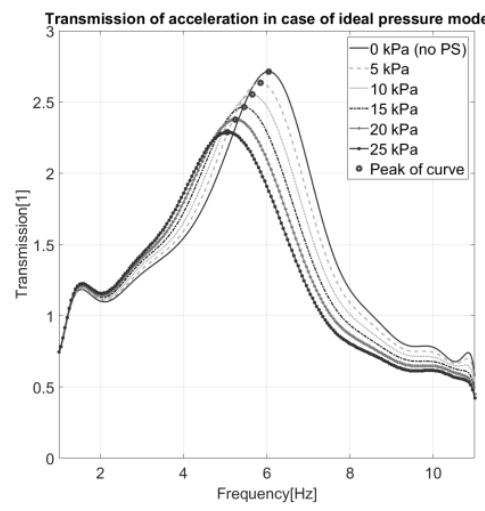

c) Ideal constant pressure mode

Fig. 4: Simulation results of transmission of acceleration

\section{Conclusion}

This article presents a derivation of the simulation model of a pneumatic spring element in interaction with mass. Its dynamical behavior is then investigated. The results show that the increase of the pressure inside the pneumatic spring element causes the value of the peak of the transmission curve smaller and to appear at a lower frequency in both working modes in the considered frequency range $[1,11] \mathrm{Hz}$. The decrease of this peak is an important factor in passenger comfort assessment. The simulation results also show that pneumatic spring element works more effectively in case of the ideal constant pressure mode and provides a more significant reduction of the peak value when the $p_{s}$ pressure is increased. This poses a requirement to improve the control system so that it works faster and more precisely for excitation with the frequency greater than $1 \mathrm{~Hz}$.

\section{Acknowledgement}

This article was written at the Technical University of Liberec, Faculty of Mechanical Engineering with the support of the Institutional Endowment for the Long Term Conceptual Development of Research Institutes, as provided by the Ministry of Education, Youth and Sports of the Czech Republic in the year 2018 .

\section{References}

Cirkl, D. (2012) Seat, patent no. 303163.

Cirkl, D. and TranXuan,T. (2016) Simulation model of seat with implemented pneumatic spring. Vibroengineering PROCEDIA, vol 7, ISSN 2345-0533, pp. 154-159.

Cirkl, D. and TranXuan,T. (2016) Simulation model of seat with implemented pneumatic spring with consideration of variable pressure in air reservoir. 32 ${ }^{\text {nd }}$ Computational Mechanics. University of West Bohemia, Pilsen.

Cirkl, D. and Hrus, T. (2013) Simulation Model of Polyurethane Foam for Uniaxial Dynamical Compression. Vibroengineering PROCEDIA, vol 1, ISSN 2345-0533, pp. 50-58. 